\title{
Binomial edge ideals with quadratic Gröbner bases
}

\author{
Marilena Crupi \\ Dipartimento di Matematica, \\ Università di Messina \\ Viale Ferdinando Stagno d'Alcontres, 31 \\ 98166 Messina, Italy \\ mcrupi@unime.it
}

\author{
Giancarlo Rinaldo \\ Dipartimento di Matematica, \\ Università di Messina \\ Viale Ferdinando Stagno d'Alcontres, 31 \\ 98166 Messina, Italy \\ giancarlo.rinaldo@tiscali.it
}

Submitted: Apr 15, 2011; Accepted: Oct 19, 2011; Published: Oct 31, 2011

Mathematics Subject Classifications: 13C05, 05C25

\begin{abstract}
We prove that a binomial edge ideal of a graph $G$ has a quadratic Gröbner basis with respect to some term order if and only if the graph $G$ is closed with respect to a given labelling of the vertices. We also state some criteria for the closedness of a graph $G$ that do not depend on the labelling of its vertex set.
\end{abstract}

\section{Introduction}

In this article a graph $G$ means a simple graph without isolated vertices, loops and multiple edges. Let $V(G)=[n]=\{1, \ldots, n\}$ denote the set of vertices and $E(G)$ the set of edges.

One of the main objects of study in combinatorial commutative algebra is the edge ideal of a graph $G$ which is generated by the monomials $x_{i} x_{j}$, where $\{i, j\}$ is an edge of $G$, in the polynomial ring $K\left[x_{1}, \ldots, x_{n}\right]$ over the field $K$. Edge ideals of a graph has been introduced by Villarreal in 1990 [16], where he studied the Cohen-Macaulay property of such ideals. Many authors have focused their attention on such ideals (see for example $[15],[9],[7],[2])$.

In 2010, binomial edge ideals were introduced in [10] and appear independently, but at the same time, also in [13]. Let $S=K\left[x_{1}, \cdots, x_{n}, y_{1}, \cdots, y_{n}\right]$ be the polynomial ring in $2 n$ variables with coefficients in a field $K$. For $i<j$, set $f_{i j}=x_{i} y_{j}-x_{j} y_{i}$. The ideal $J_{G}$ of $S$ generated by the binomials $f_{i j}=x_{i} y_{j}-x_{j} y_{i}$ such that $i<j$ and $\{i, j\}$ is an edge of $G$, is called the binomial edge ideal of $G$.

Such class of ideals is a natural generalization of the ideal of 2 -minors of a $2 \times n$-matrix of indeterminates. Really, the ideal of 2-minors of a $2 \times n$-matrix may be considered as the binomial edge ideal of a complete graph on $[n]$. Moreover the binomial edge ideal of a line graph, which can be interpreted as an ideal of adjacent minors, has been examined in [3]. The importance of this class of binomial edge ideals for algebraic statistics 
is unquestionable [10]. Indeed these ideals arise naturally in the study of conditional independence statements [4]. Many algebraic properties of binomial edge ideals in terms of properties of the underlying graph were studied in [10] and [12].

In [10], Theorem 1.1, the authors proved the following:

Theorem 1.1. Let $G$ be a graph on the vertex set $[n]$, and let $<$ the lexicographic order induced by $x_{1}>\cdots>x_{n}>y_{1}>\cdots>y_{n}$ on $S$. Then the following conditions are equivalent:

(1) The generators $f_{i j}$ of $J_{G}$ form a quadratic Gröbner basis.

(2) For all edges $\{i, j\}$ and $\{k, \ell\}$ with $i<j$ and $k<\ell$ one has $\{j, \ell\} \in E(G)$ if $i=k$, and $\{i, k\} \in E(G)$ if $j=\ell$.

The authors in [10], called a graph $G$ on $[n]$ closed with respect to the given labelling of the vertices if $G$ satisfies condition (2). The term closed graph is not standard terminology in graph theory. Nevertheless this class of graphs is related to a well-known class of graphs: the chordal graphs. A closed graph is chordal ([10]) but the converse is not true. Indeed a closed graph is a claw-free chordal graph, where by a claw we mean a graph with three different edges $e_{1}, e_{2}, e_{3}$ such that $e_{1} \cap e_{2} \cap e_{3} \neq \emptyset$.

In Theorem 1.1 the role of the lexicographic order on $S$ is fundamental. In this article we are able to state that the existence of a quadratic Gröbner basis for $J_{G}$ is not related to the lexicographic order on $S$. In fact, one of the main result in the paper implies that the closed graphs are the only graphs for which the binomial edge ideal $J_{G}$ has a quadratic Gröbner basis with respect to some term order on $S$ (Theorem 3.4). Our result underlines also the relation between binomial edge ideals and edge ideals. In fact as a consequence we obtain that $J_{G}$ has a quadratic Gröbner basis with respect to some term order $\prec$ on $S$ if and only if $\operatorname{in}\left(J_{G}\right)$ is the edge ideal of a bipartite graph with bipartition $V_{1}=\left\{x_{1}, \cdots, x_{n}\right\}$ and $V_{2}=\left\{y_{1}, \cdots, y_{n}\right\}$. The strict relation between algebraic invariants of an ideal $J$ and $\operatorname{in}(J)$ is well known (see for example [5], Chapter 15).

Furthermore Theorem 1.1 and Theorem 3.4 suggest that it would be interesting to state some criteria for the closedness of a simple graph $G$. Since the characterizations of closed graphs $G$ (see [10], [12]) depend on the labelling of $V(G)$, our aim is to state some new criteria for the closedness of a graph that do not depend on the labelling of its vertex set (Theorem 5.5 and Corollary 5.7).

We believe that by an ordering on the vertices obtained by lexicographic breadth first search and an appropriate specialization of the algorithm on chordality test (see Algorithms 2, 3 of [8] or [14]), it is possible to test the closedness of a graph as a consequence of Theorem 5.5 in linear time. But this is not the aim of this paper.

The paper is organized as follows.

Section 2 contains some preliminaries and notions that we will use in the paper.

In Section 3, we state a fundamental result that gives the motivation of an intensive study of closed graphs: we prove that the only graphs having quadratic Gröbner basis with respect to a given monomial order are the closed ones (Theorem 3.4). The statement is obtained by the construction of a special oriented graph (Definition 3.1). 
In Section 4, we introduce the notion of a linear quasi-tree simplicial complex (Definition 4.3) and we relate it with a closed graph (Proposition 4.6). Moreover we give a characterization of the closedness of a graph $G$ in terms of particular cliques of $G$ (Proposition 4.8). This result will be crucial in the sequel.

In Section 5, we analyze the behaviour of the set of facets $\mathcal{F}(\Delta(G))$ of the clique complex $\Delta(G)$ (Definition 2.1) of a graph G when $\Delta(G)$ is a linear quasi-tree (Proposition 5.1). We introduce a special subclass of the linear quasi-tree complexes: the class of closed complexes (Definition 5.2). The section contains the main results in the paper. We give a criterion for the closedness of a graph $G$ that is independent from the labelling of $V(G)$ (Theorem 5.5). We show that a graph $G$ is closed if and only if the clique complex $\Delta(G)$ is a closed complex (Corollary 5.7).

\section{Preliminaries}

In this section we recall some concepts and a notation on graphs and on simplicial complexes that we will use in the article.

Let $G$ be a simple graph with vertex set $V(G)$ and the edge set $E(G)$. Let $v, w \in V(G)$. A path $\pi$ from $v$ to $w$ is a sequence of vertices $v=v_{0}, v_{1}, \cdots, v_{t}=w$ such that $\left\{v_{i}, v_{i+1}\right\}$ is an edge of the underlying graph. A graph $G$ is connected if for every pair of vertices $v_{1}$ and $v_{2}$ there is a path from $v_{1}$ to $v_{2}$. If $G$ is directed (or digraph), that is, $G$ consists of a finite nonempty set of vertices with a prescribed collection $X$ of ordered pairs of distinct vertices, then the path is called directed, if either $\left(v_{i}, v_{i+1}\right)$ is an arrow for all $i$, or $\left(v_{i+1}, v_{i}\right)$ is an arrow for all $i$.

When we fix a given labelling on the vertices we say that $G$ is a graph on $[n]$.

Let $G$ be a graph with vertex set $[n]$. A subset $C$ of $[n]$ is called a clique of $G$ is for all $i$ and $j$ belonging to $C$ with $i \neq j$ one has $\{i, j\} \in E(G)$.

Set $V=\left\{x_{1}, \ldots, x_{n}\right\}$. A simplicial complex $\Delta$ on the vertex set $V$ is a collection of subsets of $V$ such that

(i) $\left\{x_{i}\right\} \in \Delta$ for all $x_{i} \in V$ and

(ii) $F \in \Delta$ and $G \subseteq F$ imply $G \in \Delta$.

An element $F \in \Delta$ is called a face of $\Delta$. For $F \in \Delta$ we define the dimension of $F$ by $\operatorname{dim} F=|F|-1$, where $|F|$ is the cardinality of the set $F$. A maximal face of $\Delta$ with respect to inclusion is called a facet of $\Delta$.

If $\Delta$ is a simplicial complex with facets $F_{1}, \ldots, F_{q}$, we call $\left\{F_{1}, \ldots, F_{q}\right\}$ the facet set of $\Delta$ and we denote it by $\mathcal{F}(\Delta)$. When $\mathcal{F}(\Delta)=\left\{F_{1}, \ldots, F_{q}\right\}$, we write $\Delta=\left\langle F_{1}, \ldots, F_{q}\right\rangle$.

Definition 2.1. The clique complex $\Delta(G)$ of $G$ is the simplicial complex whose faces are the cliques of $G$.

Definition 2.2. Let $\Delta$ be a simplicial complex. A facet $F \in \mathcal{F}(\Delta)$ is said to be a leaf of $\Delta$ if either $F$ is the only facet of $\Delta$, or there exists a facet $B \in \mathcal{F}(\Delta), B \neq F$, called a branch of $F$, such that $H \cap F \subseteq B \cap F$ for all $H \in \mathcal{F}(\Delta)$ with $H \neq B$. 
Observe that for a leaf $F$ the subcomplex $\Delta^{\prime}$ with $\mathcal{F}\left(\Delta^{\prime}\right)=\mathcal{F}(\Delta) \backslash F$ coincides with the restriction $\Delta_{[n] \backslash(F \backslash(B \cap F)) \text {. }}$

We finish this section by recalling the following definition from [11].

Definition 2.3. Let $\Delta$ be a simplicial complex. $\Delta$ is called a quasi-forest if there exists a labelling $F_{1}, \cdots, F_{q}$ of the facets of $\Delta$, such that for every $1<i \leq q$, the facet $F_{i}$ is a leaf of the subcomplex $\left\langle F_{1}, \cdots, F_{i}\right\rangle$. The sequence $F_{1}, \ldots, F_{q}$ is called a leaf order of the quasi-tree. A connected quasi-forest is called a quasi-tree.

\section{Quadratic Gröbner bases}

In this section we observe that the only graphs having quadratic Gröbner bases with respect to a monomial order $\prec$ are the closed graphs with respect to a labelling induced by $\prec$.

Let $G$ be a graph on the vertex set $V(G)=[n], E(G)$ its edge set and $S=$ $K\left[x_{1}, \cdots, x_{n}\right.$, $\left.y_{1}, \cdots, y_{n}\right]$.

Definition 3.1. Let $J_{G}$ be the binomial edge ideal of $G$ and let $\prec$ a term order on $S$. We define an oriented graph $G_{\prec}$ with $V\left(G_{\prec}\right)=V(G)$ and edge set

$$
E\left(G_{\prec}\right)=\left\{(i, j): x_{i} y_{j} \in \operatorname{in}_{\prec} J_{G}\right\} .
$$

Proposition 3.2. $G_{\prec}$ is an acyclic directed graph.

Proof. It is sufficient to show that every cycle in $G$ is not a directed cycle in $G_{\prec}$. Let

$$
\left\{i_{1}, i_{2}, \ldots, i_{r}\right\} \subseteq V(G)
$$

be the vertices of a cycle and suppose that $\left(i_{j}, i_{j+1}\right) \in E\left(G_{\prec}\right)$ for $j=1, \ldots, r-1$. We will show that $\left(i_{r}, i_{1}\right) \notin E\left(G_{\prec}\right)$.

By hypothesis we have that $x_{i_{j}} y_{i_{j+1}} \succ x_{i_{j+1}} y_{i_{j}}$ for $j=1, \ldots, r-1$. Since $\prec$ is a term order, then $y_{i_{3}}\left(x_{i_{1}} y_{i_{2}}\right) \succ y_{i_{3}}\left(x_{i_{2}} y_{i_{1}}\right)$ and $y_{i_{1}}\left(x_{i_{2}} y_{i_{3}}\right) \succ y_{i_{1}}\left(x_{i_{3}} y_{i_{2}}\right)$. Therefore $y_{i_{3}}\left(x_{i_{1}} y_{i_{2}}\right) \succ$ $y_{i_{1}}\left(x_{i_{3}} y_{i_{2}}\right)$ and $x_{i_{1}} y_{i_{3}} \succ x_{i_{3}} y_{i_{1}}$.

By the same argument we have that $y_{i_{4}}\left(x_{i_{1}} y_{i_{3}}\right) \succ y_{i_{4}}\left(x_{i_{3}} y_{i_{1}}\right)$ and $y_{i_{1}}\left(x_{i_{3}} y_{i_{4}}\right) \succ$ $y_{i_{1}}\left(x_{i_{4}} y_{i_{3}}\right)$. Hence $y_{i_{4}}\left(x_{i_{1}} y_{i_{3}}\right) \succ y_{i_{1}}\left(x_{i_{4}} y_{i_{3}}\right)$ and $x_{i_{1}} y_{i_{4}} \succ x_{i_{4}} y_{i_{1}}$, and so on. Finally, we will have that $x_{i_{1}} y_{i_{r}} \succ x_{i_{r}} y_{i_{1}}$.

Remark 3.3. We observe that the ideal $J_{G}$ of $S$ is multigraded if we assign the following multidegrees to the indeterminates of $S$ :

$$
\operatorname{deg}\left(x_{i}\right)=\operatorname{deg}\left(y_{i}\right)=(0, \ldots, 0,1,0, \ldots, 0) \in \mathbb{N}^{n},
$$

where the entry 1 is at the $i$-th position. Hence the only binomials of degree 2 in $J_{G}$ are the generators of $J_{G}$ up to scaling. 
Theorem 3.4. Let $G$ be a graph. The following conditions are equivalent:

(1) $G$ is closed on $[n]$;

(2) $J_{G}$ has a quadratic Gröbner basis with respect to some term order $\prec$ on $S$.

Proof. $(1) \Rightarrow(2)$. See [10], Theorem 1.1.

$(2) \Rightarrow(1)$. By Proposition $3.2 G_{\prec}$ is a directed acyclic graph. Hence there exists a labelling

$$
\omega: V\left(G_{\prec}\right) \rightarrow[n]
$$

such that for all $(i, j) \in E\left(G_{\prec}\right)$ we have that $\omega(i)<\omega(j)$. This means that $\omega$ is compatible with the orientation of $G_{\prec}$ (see for example [1], Proposition 1.4.3).

We will show that the graph $G$ is closed with respect to the labelling $\omega$.

Let $i_{1}, i_{2}, i_{3} \in V\left(G_{\prec}\right)$ such that $\omega\left(i_{1}\right)=i, \omega\left(i_{2}\right)=j, \omega\left(i_{3}\right)=k$ and let $\left\{i_{1}, i_{2}\right\}$, $\left\{i_{1}, i_{3}\right\} \in E(G)$. It follows that $\{i, j\},\{i, k\}$ are edges of $G$ with respect to the labelling $\omega$. By condition (2) of Theorem 1.1, we have to analyze the following two cases:

(a) $i<j, i<k$;

(b) $i>j, i>k$.

Case (a). Since $\omega$ is compatible with the oriented graph $G_{\prec}$, we have the following inequalities

$$
x_{i_{1}} y_{i_{2}} \succ x_{i_{2}} y_{i_{1}} \text { and } x_{i_{1}} y_{i_{3}} \succ x_{i_{3}} y_{i_{1}} .
$$

By hypothesis the $S$-polynomial

$$
S\left(f_{i_{1} i_{2}}, f_{i_{1} i_{3}}\right)=y_{i_{1}} f_{i_{2} i_{3}}=y_{i_{1}}\left(x_{i_{2}} y_{i_{3}}-x_{i_{3}} y_{i_{2}}\right)
$$

reduces to 0 . Therefore there exists a binomial $x_{i_{s}} y_{i_{t}}-x_{i_{t}} y_{i_{s}} \in J_{G}$ (see Remark 3.3) whose leading monomial divides the leading monomial of $y_{i_{1}} f_{i_{2} i_{3}}$. Suppose that $\operatorname{in}\left(f_{i_{1} i_{2}}\right)=x_{i_{2}} y_{i_{1}}$. This contradicts the first inequality in (3.1). By the same argument and the second inequality in $(3.1), \operatorname{in}\left(f_{i_{1} i_{3}}\right)$ does not divide $\operatorname{in}\left(y_{i_{1}} f_{i_{2} i_{3}}\right)$. Hence $f_{i_{2} i_{3}} \in J_{G}$ and $\{j, k\}$ is an edge of $G$ with respect to the labelling $\omega$. Case (b) follows by similar arguments.

\section{Closed graphs and linear quasi-tree complexes}

In this section we introduce the notion of a simplicial complex which is a linear quasi-tree. This class of simplicial complexes is a subclass of the quasi-forest complexes (Definition 2.3). Our aim is to underline the close link that there exists between the closed graphs and these simplicial complexes. First of all we recall the following definition ([12], Definition $2.1)$.

Definition 4.1. A graph $G$ is closed if there exists a labelling for which it is closed.

We quote the next result from ([12], Theorem 2.2). 
Theorem 4.2. Let $G$ be a graph on [n]. The following conditions are equivalent:

(1) $G$ is closed;

(2) there exists a labelling of $G$ such that all facets of $\Delta(G)$ are intervals $[a, b] \subseteq[n]$.

Moreover, if the equivalent conditions hold and the facets $F_{1}, \ldots, F_{r}$ of $\Delta(G)$ are labeled such that $\min \left(F_{1}\right)<\min \left(F_{r}\right)<\cdots<\min \left(F_{q}\right)$, then $F_{1}, \ldots, F_{r}$ is a leaf order of $\Delta(G)$.

Since a graph is closed if and only if each connected component is closed we assume from now on that the graph $G$ is connected.

Thanks to Theorem 4.2 if $G$ is a closed graph on the vertex set $[n]$ and $\Delta(G)$ is the clique complex, then we may assume that

$$
\Delta(G)=\left\langle\left[m_{1}, M_{1}\right],\left[m_{2}, M_{2}\right], \ldots,\left[m_{r}, M_{r}\right]\right\rangle,
$$

with $1=m_{1}<m_{2}<\ldots<m_{r}<n, 1<M_{1}<M_{2}<\ldots<M_{r}=n$ with $m_{i}<M_{i}$ and $m_{i+1} \leq M_{i}$, for $i \in[r]$.

Now we introduce a special subclass of the quasi-trees complexes.

Definition 4.3. A simplicial complex is a linear quasi-tree if there exists an order on the facets

$$
F_{1}, \ldots, F_{q}
$$

such that

(1) $F_{i}$ is a leaf for the subcomplex $\left\langle F_{i}, \ldots, F_{q}\right\rangle$;

(2) $F_{i+1}$ is the only branch of $F_{i}$ for all $i<q$.

Remark 4.4. Let $\Delta$ be a simplicial complex and let $\mathcal{F}(\Delta)=\left\{F_{1}, \ldots, F_{q}\right\}$ be the set of its facets. It is always possible to verify if $\Delta$ is a linear quasi tree and in the positive case it is possible to order $\mathcal{F}(\Delta)$ so that conditions (1) and (2) of Definition 4.3 are satisfied. In fact, if $\Delta$ is a linear quasi tree, then there exists a leaf $F_{i}$, that is a facet of $\Delta$ satisfying Definition 2.2. In order to determine $F_{i}$ it is sufficient to intersect the facet $F_{i}, i=1, \ldots, q$, with the other facets. Let $F_{i_{1}}$ be such a facet and let $F_{i_{2}}$ be its branch. It must be unique by (2) of Definition 4.3.

If $F_{i_{1}}$ is a leaf and $F_{i_{2}}$ is its unique branch, then we consider the subcomplex $\Delta^{\prime}=$ $\mathcal{F}(\Delta) \backslash\left\{F_{i_{1}}\right\}$ and we verify if $F_{i_{2}}$ is a leaf of $\Delta^{\prime}$ and if its branch is unique and so on. Proceeding in this way we will obtain a linear order $F_{i_{1}}, F_{i_{2}}, \ldots, F_{i_{q}}$ with respect to which $\Delta$ is a linear quasi tree.

We will show this process by the next example.

Example 4.5. Let $\Delta=\left\langle F_{1}, F_{2}, F_{3}, F_{4}\right\rangle$, with $F_{1}=\{a, b, f\}, F_{2}=\{a, e, f\}, F_{3}=\{b, c, f\}$ and $F_{4}=\{d, e, f\}$. We want to determine a order on the facet set $\mathcal{F}(\Delta)$ so that $\Delta$ is a linear quasi tree.

Consider the facet $F_{1}$. We have:

$$
F_{1} \cap F_{2}=\{a, f\}, \quad F_{1} \cap F_{3}=\{b, f\}, \quad F_{1} \cap F_{4}=\{f\} .
$$


Since $F_{1} \cap F_{2}$ and $F_{1} \cap F_{3}$ are not comparable, then $F_{1}$ is not a leaf of $\Delta$ (Definition 2.2). Consider the facet $F_{2}$. We have:

$$
F_{2} \cap F_{1}=\{a, f\}, \quad F_{2} \cap F_{3}=\{f\}, \quad F_{2} \cap F_{4}=\{e, f\} .
$$

Since $F_{2} \cap F_{1}$ and $F_{2} \cap F_{4}$ are not comparable, then $F_{2}$ is not a leaf of $\Delta$ (Definition 2.2). Now consider the facet $F_{3}$. We have:

$$
F_{3} \cap F_{1}=\{b, f\}, \quad F_{3} \cap F_{2}=\{f\}, \quad F_{3} \cap F_{4}=\{f\} .
$$

Hence $F_{1}$ is the unique branch of $F_{3}$ and consequently $F_{3}$ is a leaf of $\Delta$. Now consider the subcomplex of $\Delta: \Delta^{\prime}=\left\langle F_{1}, F_{2}, F_{4}\right\rangle$. We have:

$$
F_{1} \cap F_{2}=\{a, f\}, \quad F_{1} \cap F_{4}=\{f\} .
$$

It follows that $F_{2}$ is the unique branch of $F_{1}$ and $F_{1}$ is a leaf of $\Delta^{\prime}$. It is easy to observe that we can conclude that $\Delta$ is a linear quasi tree with respect to the following order on $\mathcal{F}(\Delta): F_{3}, F_{1}, F_{2}, F_{4}$.

From now on when we consider a simplicial complex $\Delta$ that is a linear quasi-tree we write $\Delta=\left\langle F_{1}, \ldots, F_{q}\right\rangle$ with leaf order $\left\{F_{1}, F_{2}, \ldots, F_{q}\right\}$ on the facet set. We state the following.

Proposition 4.6. Let $G$ be a graph on $[n]$. If $G$ is a closed graph, then $\Delta(G)$ is a linear quasi-tree.

Proof. From (4.1), since $G$ is closed, we may assume $\Delta(G)=\left\langle F_{1}, \ldots, F_{r}\right\rangle$, where $F_{i}=$ $\left[m_{i}, M_{i}\right]$, for $i=1, \ldots, r$.

We observe that $\left[m_{i}, M_{i}\right] \cap\left[m_{i+1}, M_{i+1}\right]=\left[m_{i+1}, M_{i}\right]$. Since $m_{i+d}>m_{i+1}$ for all $d \geq 2$, then

$$
F_{i} \cap F_{i+d}=\left[m_{i+d}, M_{i}\right] \varsubsetneqq\left[m_{i+1}, M_{i}\right] .
$$

Therefore $F_{i}$ is a leaf and $F_{i+1}$ is the unique branch for $F_{i}$.

Example 4.7. The converse of Proposition 4.6 is not true. In fact there are linear quasitrees that are not closed.

Let $V(G)=\{a, b, c, d, e, f\}$ and let $\Delta(G)=\left\langle F_{1}, F_{2}, F_{3}\right\rangle$ be the facet set of its clique complex, where $F_{1}=\{a, b, c\}, F_{2}=\{b, c, d, e\}$ and $F_{3}=\{b, e, f\}$. We can easily check that $\left\langle F_{1}, F_{2}, F_{3}\right\rangle$ is a linear quasi-tree but the subgraph induced by the vertices $\{a, b, d, f\}$ is a claw, i.e. the complete bipartite graph $K_{1,3}$. Therefore by ([10], Proposition 1.2) $G$ is not closed.

We finish this section giving a criterion for the closedness of a graph with respect to a given labelling that will be crucial in the sequel.

Let $G$ be a graph on the vertex set $V(G)=[n]$. For each vertex $j \in V(G)$ we define a partition of its neighborhood $N_{G}(j)=\{i \in[n]:\{i, j\} \in E(G)\}$ into two sets as follows:

$$
N_{G}(j)=N_{G}^{<}(j) \cup N_{G}^{>}(j),
$$


where

$$
N_{G}^{<}(j)=\{i:\{i, j\} \in E(G), i<j\}, N_{G}^{>}(j)=\{k:\{j, k\} \in E(G), j<k\} .
$$

Proposition 4.8. Let $G$ be a graph on $[n]$. The following conditions are equivalent:

(1) $G$ is closed with respect to the given order of the vertices;

(2) for all vertices $j \in V(G)$ the sets $N_{G}^{<}(j), N_{G}^{>}(j)$ are cliques of $G$.

Proof. $(1) \Rightarrow(2)$. Let $j \in V(G)$. For all $i_{1}, i_{2} \in N_{G}^{<}(j)$, by definition, we have that $\left\{i_{1}, j\right\},\left\{i_{2}, j\right\} \in E(G)$ with $i_{1}<j$ and $i_{2}<j$. Since $G$ is closed, then $\left\{i_{1}, i_{2}\right\} \in E(G)$. Hence $N_{G}^{<}(j)$ is a clique. Similarly for $N_{G}^{>}(j)$.

$(2) \Rightarrow(1)$. Let $\left\{j, k_{1}\right\},\left\{j, k_{2}\right\} \in E(G)$ with $j<k_{1}, j<k_{2}$. This implies $k_{1}, k_{2} \in N_{G}^{>}(j)$. Since $N_{G}^{>}(j)$ is a clique, then $\left\{k_{1}, k_{2}\right\} \in E(G)$. The other case follows by similar argument.

\section{Closed graphs with respect to any labelling}

In this section we give a characterization of closed graphs which does not depend on the labelling of their vertex sets. For this reason we study the clique complex $\Delta(G)$ of the simple graph $G$.

Let $\Delta=\left\langle F_{1}, \ldots, F_{r}\right\rangle$ be a simplicial complex. We set

$$
F_{i_{1}, i_{2}, \ldots, i_{s}}:=F_{i_{1}} \cap F_{i_{2}} \cap \ldots \cap F_{i_{s}}
$$

with $1 \leq i_{1}<i_{2}<\ldots<i_{s} \leq r$ and $F_{i, i}:=F_{i}$ for $i \in[r]$.

Proposition 5.1. If $\Delta=\left\langle F_{1}, \ldots, F_{r}\right\rangle$ is a linear quasi-tree, then $F_{i, j}=F_{i, i+1, \ldots, j}, 1 \leq$ $i<j \leq r$. In particular, $F_{k, \ell} \supseteq F_{i, j}$ for all $k$, $\ell$ such that $i \leq k \leq \ell \leq j$.

Proof. We proceed by descending induction on $i$, for $i<j$. If $i=j-1$ there is nothing to prove. Let $i \leq j-1$ and suppose $F_{i, j}=F_{i, i+1, \ldots, j}$. We have to prove that $F_{i-1, j}=$ $F_{i-1, i, i+1, \ldots, j}$.

Since $F_{i-1, i, j}=F_{i-1} \cap F_{i, j}=F_{i-1, i, i+1, \ldots, j}$, we need to show that

$$
F_{i-1} \cap F_{i, j}=F_{i-1, j}
$$

By definition $F_{i-1, i, j} \subseteq F_{i-1, j}$. Since $F_{i}$ is a branch of $F_{i-1}$, then $F_{i-1, j} \subseteq F_{i-1, i}$. Hence $F_{i-1, j} \cap F_{j} \subseteq F_{i-1, i} \cap F_{j}$, that is, $F_{i-1, j} \subseteq F_{i-1, i, j}$ and the assertion follows.

Denote by $\mathcal{P}=\left\{F_{i, j}: 1 \leq i \leq j \leq r\right\}$ the poset whose order is given by the inclusion and set $F_{i, j}=\emptyset$ if either $i<1$ or $j>r$. If $F, G \in \mathcal{P}$ are not comparable or $F \neq \emptyset$ or $G \neq \emptyset$, we write $F \nsim G$. 
Definition 5.2. Let $\Delta=\left\langle F_{1}, \ldots, F_{r}\right\rangle$ be a linear quasi-tree. $\Delta$ is called closed if the following properties are satisfied:

(I) $F_{i, j} \nsim F_{k, \ell}$ if $i<k, j<\ell, i, j, k, \ell \in[r]$ (incomparability);

(C) $F_{i+1, i+d}=F_{i, i+d} \cup F_{i+1, i+d+1}$ if $F_{i, i+d+1} \neq \emptyset$ with $d \geq 1$ and $i \in[r]$ (covering).

Theorem 5.3. Let $G$ be a graph on [n]. If $G$ is closed, then $\Delta(G)$ is closed.

Proof. Since, from Proposition 4.6, $\Delta(G)$ is a linear quasi-tree, we have only to prove that the facet set $\mathcal{F}(\Delta(G))=\left\{F_{1}, \ldots, F_{r}\right\}$ satisfies properties (I) and (C) in Definition 5.2.

(I). Since $G$ is closed on $[n]$, if $F_{i, j} \neq \emptyset$ and $F_{k, \ell} \neq \emptyset$, from (4.1) we have:

$$
\begin{aligned}
F_{i, j} & =F_{i} \cap F_{j}=\left[m_{i}, M_{i}\right] \cap\left[m_{j}, M_{j}\right]=\left[m_{j}, M_{i}\right], \\
F_{k, \ell} & =F_{k} \cap F_{\ell}=\left[m_{k}, M_{k}\right] \cap\left[m_{\ell}, M_{\ell}\right]=\left[m_{\ell}, M_{k}\right],
\end{aligned}
$$

with $i<j$ and $k<\ell$. We may assume $i<k$ and $j<\ell$. Hence by (4.1) $m_{j}<m_{\ell}$ and $M_{i}<M_{k}$. Therefore $M_{k} \in F_{k, \ell} \backslash F_{i, j}$ and $m_{j} \in F_{i, j} \backslash F_{k, \ell}$, that is $F_{i, j} \nsim F_{k, \ell}$.

(C). Since $F_{i, i+d+1} \neq \emptyset$ and $G$ is closed, then

$$
F_{i, i+d+1}=\left[m_{i+d+1}, M_{i}\right] \neq \emptyset .
$$

Therefore $m_{i+d+1} \leq M_{i}$, and

$$
F_{i, i+d} \cup F_{i+1, i+d+1}=\left[m_{i+d}, M_{i}\right] \cup\left[m_{i+d+1}, M_{i+1}\right]=\left[m_{i+d}, M_{i+1}\right]=F_{i+1, i+d} .
$$

To prove that $\Delta(G)$ closed implies $G$ closed we need a labelling on the vertices of $G$ for which $G$ is closed.

Lemma 5.4. Let $\Delta(G)=\left\langle F_{1}, \ldots, F_{r}\right\rangle$ be a linear quasi-tree. Set $n_{i}=\max \left\{j: F_{i, j} \neq\right.$ $\emptyset, j \in[r]\}$. Then $n_{1} \leq n_{2} \leq \cdots \leq n_{r}$ and every set $F_{i, j}$ in

$$
\mathcal{B}=\left\{F_{1,1}, \ldots, F_{1, n_{1}}, F_{2, n_{1}}, \ldots, F_{2, n_{2}}, \ldots, F_{r, r}\right\}
$$

is not empty.

Proof. Since $F_{i, n_{i}} \neq \emptyset$, then $F_{i+1, n_{i}} \neq \emptyset$ (Proposition 5.1). Hence $n_{i+1} \geq n_{i}$. Moreover, by Proposition 5.1 , we can also state that every set in $\mathcal{B}$ is not empty.

Now we are in position to state the main result in the paper.

Theorem 5.5. Let $G$ be a graph. Suppose that $\Delta(G)$ is closed. Let $F_{1}, \ldots, F_{r}$ be the leaf order of $\Delta(G)$ and consider the family

$$
\mathcal{F}=\left\{F_{i, j}^{\prime}\right\}_{F_{i, j} \in \mathcal{B}},
$$

where $\mathcal{B}$ is defined as in Lemma 5.4 and $F_{i, j}^{\prime}=F_{i, j} \backslash\left(F_{i-1, j} \cup F_{i, j+1}\right)$. Then 
(1) The family $\mathcal{F}$ is a partition of $V(G)$;

(2) $G$ is closed with respect to the following total order on the vertices: For the vertices in each $F_{i, j}^{\prime}$ we fix an arbitrary total order and set $u<v$, if $u \in F_{i, j}^{\prime}$ and $v \in F_{k, \ell}^{\prime}$ with $i<k$ or $i=k$ and $j<\ell$.

Proof. (1). First of all, we prove the following claim.

Claim 5.6. Let $F_{i, j} \neq \emptyset$ then

$$
F_{i} \cup F_{j}=\bigcup_{k=i}^{j} F_{k}=\left(\bigcup_{k=i}^{j-1} F_{i, k}\right) \cup\left(\bigcup_{k=i+1}^{j} F_{k, j}\right) \cup F_{i, j} .
$$

Proof of the Claim. Let $i \leq k \leq \ell \leq j$. Since, by assumption $F_{i, j} \neq \emptyset$, then Proposition 5.1 implies that $F_{k, \ell} \neq \emptyset$.

By condition (I) in Definition 5.2 and Proposition 5.1, $F_{k, \ell} \subseteq F_{i, j}$ if and only if $1 \leq i \leq$ $k \leq \ell \leq j \leq r$. Hence the poset $\mathcal{P}_{i j}=\left\{F_{k, \ell}: i \leq k \leq \ell \leq j\right\}$, whose partial order is given by the inclusion, is the following:

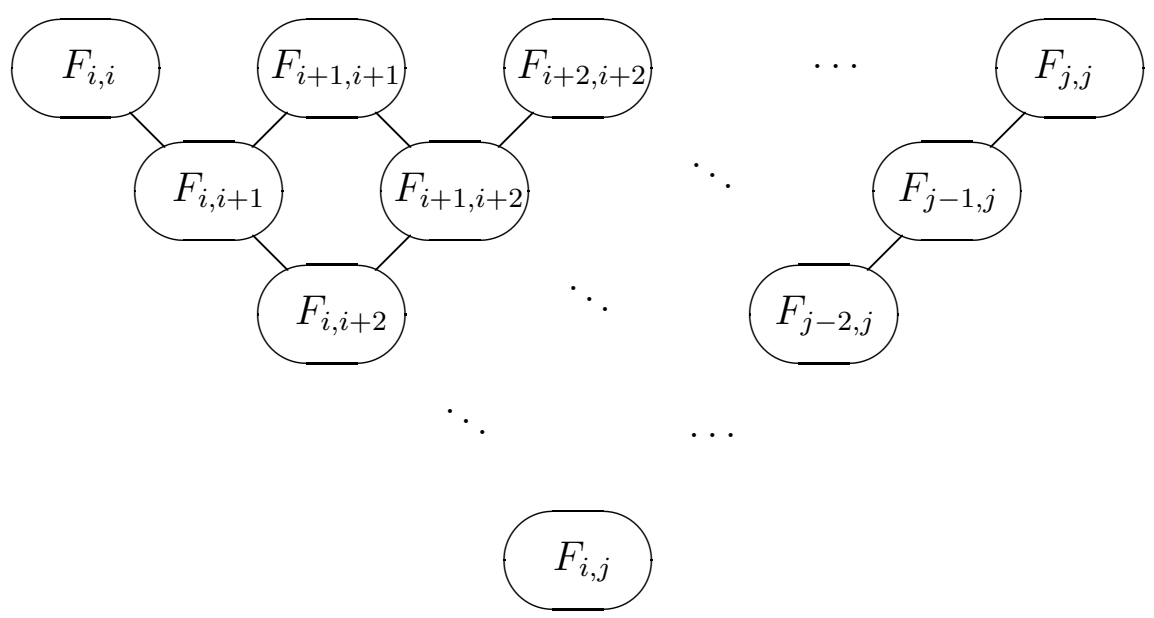

We observe that $\bigcup_{k=i}^{j} F_{k}=\bigcup_{F \in \mathcal{P}_{i, j}} F$. Since $F_{k-1, k+1} \neq \emptyset$, for $k=i+1, \ldots, j-1$, then by condition (C) we have $F_{k, k}=F_{k-1, k} \cup F_{k, k+1}$, that is

$$
\bigcup_{k=i}^{j} F_{k}=\bigcup_{F \in \mathcal{P}_{i, j}^{\prime}} F
$$

with $\mathcal{P}_{i, j}^{\prime}=\mathcal{P}_{i, j} \backslash\left\{F_{k}: k=i+1, \ldots, j-1\right\}$. By similar argument we may subtract all the redundant elements $F_{k, \ell}$ with $i<k<\ell<j$. Hence

$$
\bigcup_{k=i}^{j} F_{k}=\left(\bigcup_{k=i}^{j-1} F_{i, k}\right) \cup\left(\bigcup_{k=i+1}^{j} F_{k, j}\right) \cup F_{i, j}=F_{i} \cup F_{j},
$$


and Claim 5.6 is proved.

Let $\mathcal{P}=\left\{F_{i, j}: 1 \leq i \leq j \leq r\right\}$ be the poset induced by the inclusion. We say that an element $F_{i, j} \in \mathcal{P}$ is an inner element if $F_{i-1, j+1} \in \mathcal{P}$ and $F_{i-1, j+1} \neq \emptyset$. Otherwise an element of $\mathcal{P}$ is said to be border element.

We observe that the border elements are exactly the elements of $\mathcal{B}$ described in Lemma 5.4 , and

$$
V(G)=\bigcup_{F_{i, j} \in \mathcal{B}} F_{i, j} .
$$

In fact if $v \in V(G)$, then $v \in F_{k, k} \in \mathcal{F}(\Delta(G))$. If $F_{k, k} \in \mathcal{B}$ we have nothing to prove. Suppose $F_{k, k} \notin \mathcal{B}$ then $F_{k-1, k+1} \neq \emptyset$ and, since $\Delta(G)$ is closed by property $(C)$ $F_{k, k}=F_{k-1, k} \cup F_{k, k+1}$. We may assume $v \in F_{k-1, k}$. If $F_{k-1, k} \notin \mathcal{B}$ applying the same argument after a finite number of steps we obtain $v \in F_{i, j} \in \mathcal{B}$. If we remove the redundant elements in (5.1) we obtain

$$
V(G)=\bigcup_{F_{i, j} \in \mathcal{B}} F_{i, j}^{\prime}
$$

where $F_{i, j}^{\prime}=F_{i, j} \backslash\left(F_{i-1, j} \cup F_{i, j+1}\right)$. We observe the following

$$
\text { if } v \in F_{i, j}^{\prime} \text {, then } v \in F_{k} \text { if and only if } k=i, \ldots, j \text {. }
$$

This assertion can be deduced from the structure of the poset $\mathcal{P}$. For sake of completeness we give a direct proof. Since $v \in F_{i, j}^{\prime}$ then $v \in F_{i, j}$ and by Proposition 5.1, $v \in F_{k}$ with $k=i, \ldots, j$. Suppose that $v \in F_{\ell}$, with $\ell>j$. Then $v \in F_{i} \cap F_{\ell}=F_{i, \ell}$. Therefore $v \in F_{i, \ell} \subsetneq F_{i, j}$ and this is a contradiction since $v \in F_{i, j} \backslash F_{i, j+1}$ and $F_{i, j+1} \supseteq F_{i, \ell}$.

By (5.2), it easily follows that

$$
\mathcal{F}=\left\{F_{i, j}^{\prime}\right\}_{F_{i, j} \in \mathcal{B}}
$$

is a partition of $V(G)$.

(2). We prove that $G$ is closed with respect to the labelling induced by the ordering defined in the statement. By Proposition 4.8 it is sufficient to prove that for every $v \in V(G)$, $N_{G}^{<}(v), N_{G}^{>}(v) \in \Delta(G)$. Since $v \in V(G)$, then $v \in F_{i, j}^{\prime} \in \mathcal{F}$. We claim that $N_{G}^{>}(v) \subseteq F_{j}$, $N_{G}^{<}(v) \subseteq F_{i}$.

Let $\{v, w\} \in E(G)$ with $v<w$, we want to prove that $\{v, w\} \subseteq F_{j}$. Since $v \in F_{i j}^{\prime}$ by (5.2) the only cliques containing $v$ are $F_{i}, \ldots, F_{j}$. Therefore, since $\{v, w\}$ is contained in a clique of $G$, then $\{v, w\} \subseteq F_{i} \cup F_{i+1} \cup \ldots \cup F_{j}$. By Claim $5.6\{v, w\} \subseteq F_{i} \cup F_{j}$. Since $v<w$, we have the following cases:

(a) $w \in F_{i, j}^{\prime}$;

(b) $w \in F_{k, \ell}^{\prime}$, with $k>i$;

(c) $w \in F_{k, \ell}^{\prime}$, with $k=i$ and $\ell>j$.

(a). Obvious. (b). If $w \in F_{k, \ell}^{\prime}$ with $k>i$, then we have that $w \notin F_{i}$, by (5.2). Hence $w \in F_{j}$. (c). If $w \in F_{i, \ell}^{\prime}$ with $\ell>j$, then we have that $w \in F_{j}$, by (5.2).

By the same argument we prove that $N_{G}^{<}(v) \subset F_{i}$. 
Corollary 5.7. Let $G$ be a graph. The following conditions are equivalent:

(1) The graph $G$ is closed on $[n]$;

(2) the clique complex $\Delta(G)$ is closed;

(3) the binomial edge ideal $J_{G}$ has a quadratic Gröbner basis.

Proof. The equivalence follows from Theorems 5.3, 5.5 and 3.4.

\section{Acknowledgements}

The authors wish to thank Prof. Jürgen Herzog for much useful advice and suggestions. We are also grateful for the referee's careful reading and useful comments.

\section{References}

[1] J. Bang-Jensen and G. Gutin, Digraphs: Theory, Algorithms and Applications, Springer, 2007.

[2] M. Crupi, G. Rinaldo N.Terai, Cohen-Macaulay edge ideals whose height is half of the number of vertices, Nagoya Math. J. 201 (2011) 117-131.

[3] P. Diacons, D. Eisenbud, B. Sturmfels, Lattice walks and primary decomposition, Mathematical Essays in honor of Giancarlo Rota, Birhäuser, Boston, Cambridge, MA, 1998, 173-193.

[4] M. Drton, B. Sturmfels, S. Sullivan, Lectures on Algebraic statistics, Birhäuser, Boston, Cambridge, 2009.

[5] D. Eisenbud. Commutative algebra, with a view towards algebraic geometry, Graduate Texts Math., 150, Springer 1995.

[6] D. Eisenbud, B. Sturmfels, Binomial ideals, Duke Math. J. 84 (1996) 1-45.

[7] S. Faridi, The facet ideal of a simplicial complex, Manuscripta Math. 109 (2002) $159-174$.

[8] M. Habib, R. McConnel, C. Paul, L.Viennot, Lex-BFS and partition refinement, with applications to transitive orientation, interval graph recognition, and consecutive one testing, Theoretical Computer Science, 234 (2000) 59-84

[9] J. Herzog, T. Hibi, Distributive lattices, bipartite graphs and Alexander duality, J. Alg. Combin. 22 (2005) 289-302.

[10] J. Herzog, T. Hibi, F. Hreinsdottir, T. Kahle, J. Rauh, Binomial edge ideals and conditional independence statements, Advances in Applied Mathematics, 45 (2010) 317-333

[11] J. Herzog, T. Hibi. Monomial ideals, Graduate texts in Matematics, Springer, 2010.

[12] J. Herzog, V. Ene, T. Hibi, Cohen-Macaulay binomial edge ideals, arXiv.org: 1004.0143v1 [Math.AC], 2010. 
[13] M. Ohtani, Graphs and ideals generated by some 2-minors, to appear in Comm. Alg.

[14] D. Rose, G. Lueker, R. E. Tarjan, Algorithmic aspects of vertex elimination on graphs, SIAM Journal on Computing, 5 (1976) 266-283.

[15] A. Simis, W. Vasconcelos, R.H. Villarreal, On the ideal theory of graphs, J. Algebra 167 (1994) No.2 389-416.

[16] R.H. Villarreal, Cohen-Macaulay graphs, Manuscripta Math. 66 (1990) 277-293.

[17] R.H. Villarreal. Monomial algebras. Pure and Applied Mathematics. Marcel Dekker, New York/Basel, 2001. 